\title{
Molecular Polaritons Generated from Strong Coupling between CdSe Nanoplatelets and a Dielectric Optical Cavity
}

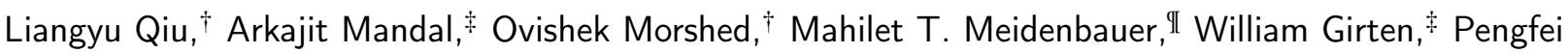 \\ Huo, ${ }^{*,+, \dagger}$ A. Nickolas Vamivakas, ${ }^{*}, \dagger, \S$ and Todd D. Krauss ${ }^{*,+, \dagger, \pi}$ \\ $\dagger$ Institute of Optics, University of Rochester, Rochester, New York, 14627, USA \\ $\$$ Department of Chemistry, University of Rochester, Rochester, New York, 14627, USA \\ IIMaterials Science Program, University of Rochester, Rochester, New York 14627, USA \\ $\S$ Department of Physics \& Astronomy, University of Rochester, Rochester, New York 14627, USA
}

E-mail: pengfei.huo@rochester.edu; nick.vamivakas@rochester.edu; krauss@chem.rochester.edu

\section{Abstract}

We demonstrate the formation of CdSe nanoplatelet (NPL) exciton-polaritons in a distributed bragg reflector (DBR) cavity. The molecule-cavity hybrid system is in the strong coupling regime with an $83 \mathrm{meV}$ Rabi splitting, characterized from angle-resolved reflectance and photoluminescence measurements. Mixed quantum-classical dynamics simulations are used to investigate the polariton photophysics of the hybrid system by treating the electronic and photonic degrees of freedom (DOF) quantum mechanically, and the nuclear phononic DOF classically. Our numerical simulations of the angle-resolved photoluminescence (PL) agree extremely well with the experimental data, providing a fundamental explanation of the asymmetric intensity distribution of the upper and lower polariton branches. Our results also provide mechanistic insights into the importance of phonon-assisted non-adiabatic transitions among polariton states which are reflected in the various features of the PL spectra. This work proves the feasibility of coupling nanoplatelet electronic states with the photon states of a dielectric cavity to form a hybrid system and provides a new platform for investigating cavity-mediated physical and chemical processes.

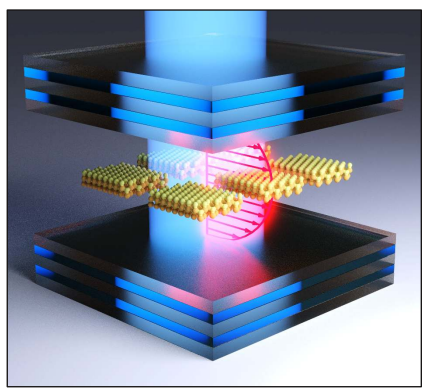

Introduction. Coupling electronic states of molecules or nanoparticles to the quantized radiation field inside an optical cavity creates a set of new photon-matter hybrid excitations, called polaritons. ${ }^{1-4}$ As opposed to atoms, the vibrational modes of molecules provide new degrees of freedom to mediate the quantum transduction between electronic and photonic states, offering new paradigms for quantum materials and chemical science. For example, strongly coupling molecules to an optical cavity has yielded great promise to manipulate chemical reactions in a general and transformative way. ${ }^{1-4}$ Through quantum light-matter interactions, the curvatures of these polaritonic potential energy surfaces can be engineered by tuning the frequency of the quantized radiation field ${ }^{5-7}$ or the coupling strength of light-matter interactions,, 5 thus opening up new possibilities to control chemical reactions by changing the fundamental properties of the quantized cavity field. $5,6,8,9$

For each molecular electronic state coupled to the cavity field, two new light-matter hybrid states are created, which are commonly referred to as the upper and lower polariton states. In addition, there is a dense manifold of states in between these two polariton states, which have predominantly of electronic character, known as dark states. ${ }^{10}$ Transitions between the dark states and the bright states induce rich polariton photophysics and novel quantum effects, such as enabling polariton lasing, ${ }^{11}$ facilitating an excitonpolariton Bose-Einstein condensate, ${ }^{12}$ or decoupling the electronic states from phonons, thus significantly enhancing the electronic coherence lifetime. ${ }^{13}$ These phenomena have been primarily investigated by forming molecular polaritons through coupling organic molecules with optical cavities. ${ }^{12-14}$ However, funda- 
mental limitations of using organic molecules for generating polaritons are their short lifetime of electronic excitations and their relatively small transition dipoles that limits the magnitude of light-matter interactions.

With recent advances in nanoparticle synthesis, colloidal nanoplatelets (NPLs) have started to draw attention due to their strong interaction with light and welldefined thickness that virtually eliminates inhomogeneous size contributions to optical spectra. ${ }^{15-19}$ Similar to 2D semiconductor quantum wells, NPLs have bulk-like properties in the transverse dimension, but exhibit quantum confinement in the longitudinal direction. ${ }^{20}$ The exceptionally narrow absorption and fluorescence linewidths of NPLs (compared to their spherical cousins colloidal quantum dots), and their exceptionally large oscillator strengths make these semiconducting NPLs promising candidates for achieving a strong light-matter coupling. Indeed NPLs have recently been incorporated into resonant photonic structures. ${ }^{17,18,21}$ Challenges for integrating colloidal NPLs into optical cavities include complex sample preparation ${ }^{17}$ or the use of plasmonic structures with a relatively low Q-factor. ${ }^{18,21}$ Importantly, the fundamental photophysical properties of the polariton states in the NPL-cavity hybrid system have not been carefully investigated.

In our work, we demonstrate the formation of exciton-polariton states resulting from the strong coupling between $\mathrm{CdSe}$ nanoplatelets and a metaldielectric hybrid cavity, depicted in Fig. 1. Angleresolved reflectance and photoluminescence measurements confirm that the system is in the strong coupling regime with an 83 meV Rabi splitting. Direct quantum dynamics simulations are used to investigate the polariton photophysics in a model Hamiltonian that includes cavity loss and phonon-assisted non-adiabatic transitions. Our results explain the universally observed asymmetric intensity distribution of the upper and lower polariton branches and underscore the importance of phonon-induced non-adiabatic transitions between polaritons. Importantly, herein we demonstrate the feasibility of generating exciton-polaritons from strong coupling between NPL excitons and a distributed bragg reflector (DBR) photonic cavity, as well as provide a detailed mechanistic understanding of the polariton photophysics from angle-resolved spectra. The system demonstrated in this work has the potential as a general platform for investigating polariton chemistry as well as for exploring transduction of quantum states among various physical degrees of freedom.

Experimental Setup. CdSe NPL synthesis was adapted from recent synthesis methods with minimal (a)

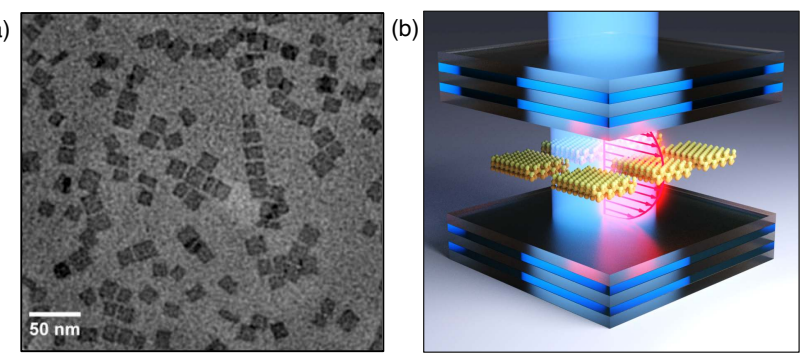

(c)

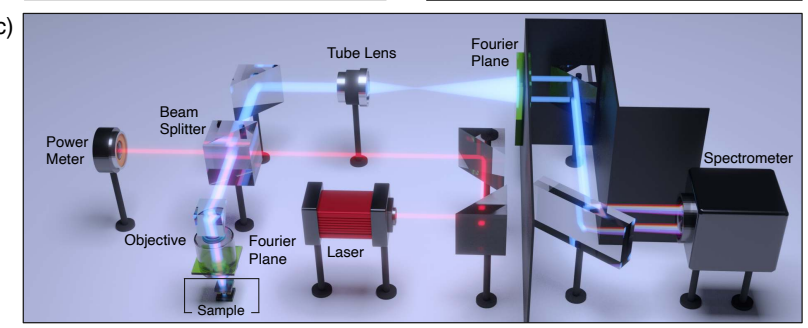

Figure 1: (a) TEM image of the synthesized nanoplatelets. (b) Illustration of the NPL-cavity hybrid system. (c) Schematic illustration of the apparatus for single-shot Fourier plane spectroscopy. Green planes stand for the Fourier plane and the conjugated Fourier plane.

adjustments. ${ }^{15,22}$ Fig. 1a presents a transmission electron microscope (TEM) image of the NPLs which have an average lateral dimension of $16 \pm 2.8 \mathrm{~nm}$ by $16 \pm$ $2.1 \mathrm{~nm}$ with thickness $\approx 1.2 \mathrm{~nm}$, corresponding to a thickness of 4.5 monolayers (MLs). Typical absorption and photoluminescence (PL) spectra of these NPLs are shown in Fig. 2a. The NPLs exhibit a well-defined heavy-hole transition peak centered at $512 \mathrm{~nm}(2.43$ $\mathrm{eV})$ and a light-hole transition peak centered at 481 $\mathrm{nm}(2.58 \mathrm{eV})$. The synthesized NPLs show an emission maximum at $512 \mathrm{~nm}(2.43 \mathrm{eV})$ with $\mathrm{FWHM} \approx 9.5 \mathrm{~nm}$ $(45 \mathrm{meV})$. A weak peak around $544 \mathrm{~nm}(2.28 \mathrm{eV})$ in the emission spectrum corresponds to residual 5.5 ML NPLs.

The optical cavity is formed by encapsulating a layer of NPLs in a polymer matrix between two highly reflective mirrors, as schematically illustrated in Fig. 1b. The bottom mirror is a distributed Bragg reflector (DBR) with 10 pairs of $\mathrm{SiO}_{2}$ and $\mathrm{SiN}_{x}$. The DBR is fabricated through plasma-enhanced chemical vapor deposition of alternating layers of $\mathrm{SiO}_{2}\left(\mathrm{SiH}_{4} / \mathrm{N}_{2} \mathrm{O}\right.$ gas $)$ and $\mathrm{SiN}_{x}\left(\mathrm{SiH}_{4} / \mathrm{NH}_{3} / \mathrm{N}_{2}\right.$ gas $)$ on top of a silicon substrate. Polymethyl methacrylate (PMMA) is used as the host material to form the cavity layer. A composite solution of NPLs and PMMA is spun-coat onto the DBR. The spin speed is controlled to result in a PMMA thickness of which the fundamental cavity mode lies around 4.5 ML emission $(\approx 2.43 \mathrm{eV})$. A $40 \mathrm{~nm}$ thick silver layer is then grown by physical vapor deposition (PVD) as the top mirror. The thickness of the silver 

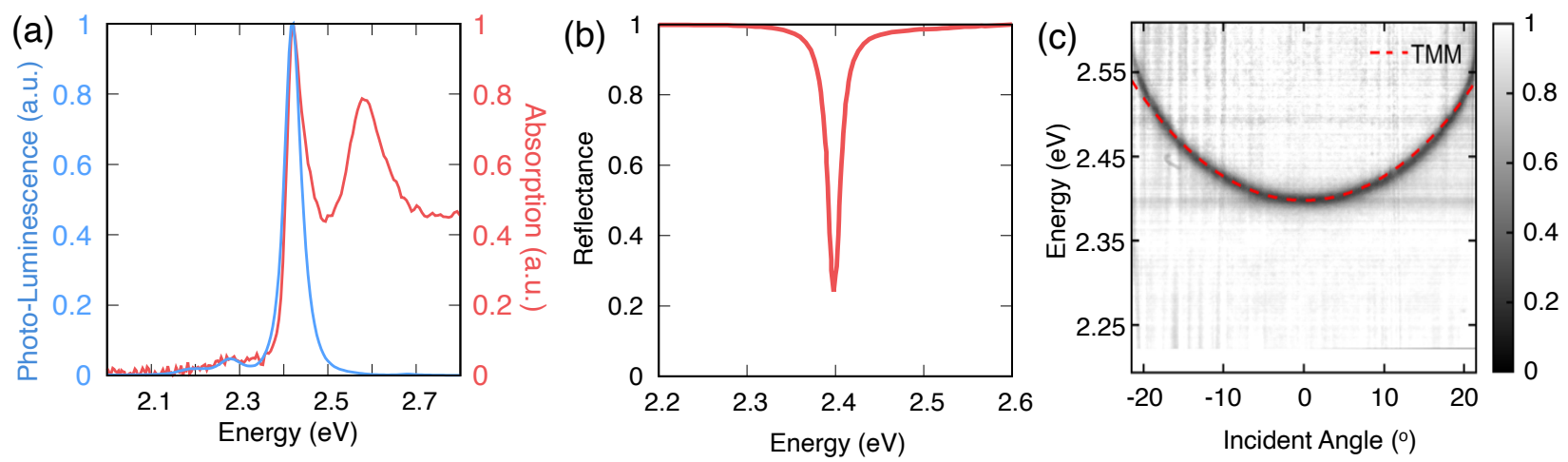

Figure 2: Characterization of the CdSe nanoplatelets and the empty cavity. (a) Absorption and photoluminescence spectra of the NPL sample in hexane at room temperature. (b) Central linecut of the angle-resolved reflectance spectrum at normal incident angle. (c) Angle-resolved reflectance spectrum of the confined cavity photon mode. Red dashed curve represents transfer-matrix method simulation of an empty cavity with corresponding thickness.

mirror is carefully selected to simultaneously result in a high Q-factor, and also enable sufficient off-resonant pump (405 nm) laser excitation. Finally, another layer of PMMA without NPLs is coated on the sample right after the PVD process to prevent silver oxidization.

A Fourier space spectroscopy system is set up to measure angle-resolved optical spectra, as shown in Fig. 1c where the back focal plane of the objective is relayed to the spectrometer's entrance slit via a tube lens. The angle information is transferred to the spatial coordinates on the slit. We use a 60X/0.7 NA objective to focus the excitation light beam and collect the output where the corresponding collection angle range is within $\pm 44^{\circ}$. We also examine a reference cavity with only PMMA (i.e. no CdSe NPLs present) using white light reflectance measurement, with the corresponding angle-resolved reflectance spectrum shown in Fig. 2c, where the parabolic shape dispersion of the cavity resonance lies as the dark region of the intensity map.

The planar DBR cavity confines the photon field in the $\mathrm{z}$ direction. For a given probing angle $\theta$ (relative to the normal vector of the cavity mirror, i.e., $\mathrm{z}$ direction), the photon energy is $E_{\mathrm{ph}}(\theta)=\frac{\hbar}{n_{\mathrm{c}}} c \sqrt{k_{\perp}^{2}+k_{\|}^{2}}=$ $\frac{\hbar}{n_{\mathrm{c}}} c \cdot k_{\perp} \sqrt{1+\tan ^{2} \theta}$, where $c$ is the speed of the light, $n_{\mathrm{c}}$ is the reflective index inside the cavity, $\hbar k_{\|}$is the in-plane momentum of photon, $\hbar k_{\perp}$ is the momentum for the quantized $\mathrm{z}$ direction, and $\tan \theta=k_{\|} / k_{\perp}$. When $\theta=0, E_{\mathrm{ph}}(0)=\hbar \omega_{\mathrm{c}}=\hbar c k_{\perp} / n_{\mathrm{c}}$, where $\omega_{\mathrm{c}}$ is the photon frequency of the quantized direction (z direction) in the cavity. Hence, in the regime of small probe angle, cavity photon energy can be approximated as

$$
E_{\mathrm{ph}}(\theta) \approx \hbar \omega_{\mathrm{c}}\left(1+\frac{1}{2} \tan ^{2} \theta\right) \equiv \hbar \omega_{\theta}
$$

The observed cavity dispersion is in a good agreement with the expected transfer-matrix simulation results (red dashed curve in Fig. 2c), in which the cavity resonance energy dispersion $E_{\mathrm{ph}}(\theta)$ as a function of incident angle is expressed in Eq. (1). In this particular cavity, $\hbar \omega_{\mathrm{c}}$ is estimated as $2.40 \mathrm{eV}$ from the central linecut, as shown in Fig. 2c. Its linewidth is estimated to be $8.9 \mathrm{meV}$, leading to a quality factor, $Q=\gamma_{\mathrm{c}} / 2 \omega_{\mathrm{c}} \approx 270$, where $\gamma_{\mathrm{c}}$ is the cavity decay rate.

Theoretical Model. The model Hamiltonian considered in this work is based on the Holstein-TavisCummings (HTC) Hamiltonian 6,11,12,23,24

$$
\hat{H}=\hat{H}_{\mathrm{NPL}}+\hat{H}_{\mathrm{ph}}+\hat{H}_{\mathrm{I}}+\hat{H}_{\mathrm{L}},
$$

where $\hat{H}_{\mathrm{NPL}}$ describes $N$ independent nanoplatelets, $\hat{H}_{\mathrm{ph}}$ is Hamiltonian for the quantized cavity mode, $\hat{H}_{\mathrm{I}}$ describes the matter-cavity interactions (between $\hat{H}_{\mathrm{NPL}}$ and $\hat{H}_{\mathrm{ph}}$ ), and $\hat{H}_{\mathrm{L}}$ describes a classical continuous-wave (CW) laser interacting with the nanoplatelets.

The matter Hamiltonian $\hat{H}_{\mathrm{NPL}}$ is modeled as a sum of $N$ identical, non-interacting nanoplatelets. For $i=$ $[1, N]$, each NPL is modeled as a three-level system, with the the ground state $\left|g_{i}\right\rangle$ with the reference electronic energy $E_{g}=0$, the heavy-hole exciton state $\left|x_{i}\right\rangle$ with the energy $E_{x}$, and the high-lying exciton state $\left|\tilde{x}_{i}\right\rangle$ with the energy $E_{\tilde{x}}$. In our model, we do not include the light-hole exciton, because that the light-hole state is decoupled from the initially occupied high-lying excited manifold (as evidenced by no PL emission for these light-hole exciton state in Fig. 2a.) Further, we approximate the continuous manifold of the high-lying exciton states with one state in resonance with the pump CW laser. The NPL matter Hamiltonian in the single 
excited manifold is expressed as

$$
\begin{gathered}
\hat{H}_{\mathrm{NPL}}=E_{G}|G\rangle\langle G|+\sum_{i=1}^{N}\left(E_{x}\left|X_{i}\right\rangle\left\langle X_{i}\left|+E_{\tilde{x}}\right| \tilde{X}_{i}\right\rangle\left\langle\tilde{X}_{i}\right|\right) \\
+\sum_{i=1}^{N} \sum_{k}\left[\frac{P_{k, i}^{2}}{2}+\frac{1}{2} \omega_{k, i}^{2}\left(R_{k, i}-\sqrt{\frac{2 \hbar S_{k, i}}{\omega_{k, i}}}\left|X_{i}\right\rangle\left\langle X_{i}\right|\right)^{2}\right]
\end{gathered}
$$

where $|G\rangle \equiv\left|g_{1}, g_{2}, . . g_{N}\right\rangle$ is the ground state of $N$ nanoplatelets with the reference electronic energy $E_{G}=$ $0,\left|X_{i}\right\rangle \equiv\left|g_{1}, g_{2}, \ldots x_{i}, \ldots g_{N}\right\rangle$ is the single excited state of the NPLs (where the $i_{\text {th }}$ NPL is excited) with energy $E_{x}$. Likewise, $\Delta U_{\tilde{X}}$ is the energy gap between a highlying excited state $\left|\tilde{X}_{i}\right\rangle \equiv\left|g_{1}, g_{2}, \ldots \tilde{x}_{i}, \ldots g_{N}\right\rangle$ of the $i_{\text {th }}$ nanoplatelet and the ground-state $|G\rangle$. Further, $P_{k, i}$ and $R_{k, i}$ are the momentum and position of the $k_{\text {th }}$ phonon mode in the $i_{\text {th }}$ NPL, with a frequency of $\hbar \omega_{k, i}$ that are coupled to the state $\left|X_{i}\right\rangle$ with the Huang-Rhys factor $S_{\alpha, i}$. The corresponding phonon reorganization energy is $\lambda_{\alpha, i}=\hbar \omega_{\alpha, i} S_{\alpha, i}$, and the reorganization energy for each individual NPL (for any $i$ ) is

$$
\lambda=\sum_{\alpha} \lambda_{\alpha, i}=\hbar \omega_{\alpha, i} S_{\alpha, i}
$$

The details of the electronic-phonon couplings are provided in the Supporting Information.

The NPL is driven by a continuous wave (CW) laser, which effectively couples the high-lying exciton state $\left|\tilde{X}_{i}\right\rangle$ to the $|G\rangle$ state through

$$
\hat{H}_{\mathrm{L}}=g_{\mathrm{L}} \sum_{i=1}^{N}\left(\left|\tilde{X}_{i}\right\rangle\langle G|+| G\rangle\left\langle\tilde{X}_{i}\right|\right) \cos \left(\omega_{\mathrm{L}} t\right)
$$

where $g_{\mathrm{L}}$ describes the laser-NPL coupling strength. In this work, we assume that the most significantly occupied high-lying exction manifold is the one that has $E_{\tilde{x}}=\hbar \omega_{\mathrm{L}}$, hence we only use one $\left|\tilde{x}_{i}\right\rangle$ state to model this manifold.

The quantized photon field Hamiltonian corresponding to $E_{\mathrm{ph}}(\theta)$ (in Eq. 1) is expressed as

$$
\hat{H}_{\mathrm{ph}}=\left(\hat{a}_{\theta}^{\dagger} \hat{a}_{\theta}+\frac{1}{2}\right) \cdot \hbar \omega_{\theta},
$$

where $\hat{a}_{\theta}^{\dagger}$ and $\hat{a}_{\theta}$ are the photonic creation and annihilation operators associated with the frequency $\hbar \omega_{\theta}=$ $\hbar \omega_{\mathrm{c}}\left(1+\frac{1}{2} \tan ^{2} \theta\right)$. In this work, we treat $\theta$ as a fixed parameter instead of a dynamical variable. ${ }^{12}$

The matter-cavity interaction term $\hat{H}_{I}$ describes the light-matter interaction within the rotating wave- approximation (RWA) as follows

$$
\hat{H}_{\mathrm{I}}=\sum_{i} g_{\mathrm{c}}\left(\hat{a}_{\theta}^{\dagger}|G\rangle\left\langle X_{i}\left|+\hat{a}_{\theta}\right| X_{i}\right\rangle\langle G|\right),
$$

where $g_{\mathrm{c}}=\sqrt{\frac{\hbar \omega_{\mathrm{c}}}{2 \varepsilon V_{\mathrm{c}}}} \hat{\mu}_{i} \cdot \hat{\mathbf{e}}$ is the matter-cavity coupling strength, with $\hat{\mu}_{i}$ as the transition dipole operator for the $i_{\text {th }}$ NPL, and $\hat{\mathbf{e}}$ is the vector field polarization direction.

The model parameters used in this Hamiltonian are directly mapped from the experimentally measured spectra. A brief summary of these parameters are provided in Table 1.

Table 1: Parameters used in the HTC model.

\begin{tabular}{cccc}
\hline \hline$N$ & $E_{x}^{0}(\mathrm{eV})$ & $\omega_{\mathrm{L}}(\mathrm{eV})$ & $g_{\mathrm{L}}(\mathrm{meV})$ \\
\hline 30 & 2.45 & 3.0 & 0.27 \\
\hline \hline
\end{tabular}

Polariton States and Strong Couplings. The polaritonic Hamiltonian is defined as

$$
\hat{H}_{\mathrm{pl}}(\mathbf{R}, \boldsymbol{\theta})=\left(\hat{H}_{\mathrm{NPL}}-\sum_{k, i} \frac{\hat{P}_{k, i}^{2}}{2}\right)+\hat{H}_{\mathrm{ph}}+\hat{H}_{\mathrm{I}},
$$

where $\mathbf{R} \equiv\left\{R_{k, i}\right\}$ and $\hat{H}_{\mathrm{pl}}(\mathbf{R}, \boldsymbol{\theta})$ parametrically depends on phonon coordinates $\mathbf{R}$. The polariton states and the polariton energy are defined through the eigen-equation

$$
\left.\hat{H}_{\mathrm{pl}}(\mathbf{R}, \theta)|\alpha(\mathbf{R}, \theta)\rangle=E_{\alpha}(\mathbf{R}, \theta)\right)|\alpha(\mathbf{R}, \theta)\rangle,
$$

where the polariton states should be viewed as the "adiabatic" states whose character parametrically depends upon $\mathbf{R}$ and $\theta$.

At the fixed ground state equilibrium geometries $(\mathbf{R}=0)$, the model reduces to the Tavis-Cummings (TC) Hamiltonian, and the symmetry of the problem allows one to define the collective bright state $|B\rangle=$ $\frac{1}{\sqrt{N}} \sum_{i}\left|X_{i}\right\rangle$, where the NPL-cavity coupling is expressed as $\hat{H}_{\mathrm{I}}=\sqrt{N} g_{\mathrm{c}}\left(\hat{a}_{\theta}^{\dagger}|G\rangle\left\langle B\left|+\hat{a}_{\theta}\right| B\right\rangle\langle G|\right)$. This further allows one to analytically express the lowest two polariton branches of $\hat{H}_{\mathrm{pl}}(\mathbf{R}=\mathbf{0}, \boldsymbol{\theta})$ as follows

$$
\begin{aligned}
E_{ \pm}(\theta)= & \frac{1}{2}\left[E_{X}^{0}+\hbar \omega_{\mathrm{c}}\left(1+\frac{1}{2} \tan ^{2} \theta\right)\right] \\
& \pm \frac{1}{2} \sqrt{\left[\hbar \omega_{\mathrm{c}}\left(1+\frac{1}{2} \tan ^{2} \theta\right)-E_{X}^{0}\right]^{2}+4 N g_{\mathrm{c}}^{2}}
\end{aligned}
$$

where $E_{X}^{0}=E_{X}+\lambda$ is the vertical Franck-Condon excitation energy between $\left|X_{i}\right\rangle$ and $|G\rangle$ at $\mathbf{R}=0, \lambda$ is the reorganization energy defined in Eq. 4. These two polariton states are commonly referred to as the upper and lower polariton branches, which can be directly 
measured from angle-resolved reflectance or absorption. The light-matter detuning is defined as

$$
\Delta E(\theta)=\omega_{\mathrm{c}}\left(1+\frac{1}{2} \tan ^{2} \theta\right)-E_{x}^{0} .
$$

Under the resonant condition (a specific incident angle $\left.\theta_{0}\right)$ where the light-matter detuning $\Delta E\left(\theta_{0}\right)$ equals to 0 , the energy difference between $E_{+}\left(\theta_{0}\right)$ and $E_{-}\left(\theta_{0}\right)$ is

$$
\hbar \Omega_{\mathrm{R}}=E_{+}\left(\theta_{0}\right)-E_{-}\left(\theta_{0}\right)=2 \sqrt{N} g_{\mathrm{c}}
$$

which is commonly referred to as the Rabi splitting. It is clear that the Rabi splitting is proportional to $\sqrt{N}$, i.e., the more NPLs effectively coupled to the cavity, the larger $\Omega_{\mathrm{R}}$ is. The strong coupling criterion of forming the cavity polariton is achieved when the Rabi splitting $\Omega_{\mathrm{R}}$ is larger than the combined decay rates of photon $\left(\gamma_{c}\right)$ and exciton $\left(\gamma_{x}\right)$, such that

$$
\hbar \Omega_{\mathrm{R}}>\hbar \gamma_{\mathrm{c}}+\hbar \gamma_{x} .
$$

Since the lifetime of the NPL exciton is much longer than the cavity lifetime of photon ( $\left.\hbar \gamma_{x} \ll \hbar \gamma_{\mathrm{c}}\right)$, the condition for the matter-cavity hybrid system to achieve strong coupling is dominated by the photonic decay processes.

Moreover, the corresponding polariton eigenstates for $\hat{H}_{\mathrm{pl}}(\mathbf{R}=0, \theta)$ are

$$
\begin{aligned}
& |+\rangle=\sin \phi \cdot\left[\frac{1}{\sqrt{N}} \sum_{i}\left|X_{i}, 0\right\rangle\right]+\cos \phi|G, 1\rangle \\
& |-\rangle=\cos \phi \cdot\left[\frac{1}{\sqrt{N}} \sum_{i}\left|X_{i}, 0\right\rangle\right]-\sin \phi|G, 1\rangle
\end{aligned}
$$

where the matter-cavity mixing angle is $\phi=$ $\frac{1}{2} \tan ^{-1}\left(\frac{2 \hbar \sqrt{N} g_{\mathrm{c}}}{\Delta E(\theta)}\right)$, with the matter-cavity detuning $\Delta E(\theta)$ defined in Eq. 11. The following Hopfield coefficients indicate the character of polariton states

$$
\begin{aligned}
|X(\theta)|^{2} & =|\sin \phi|^{2}=\frac{1}{2}\left(1+\frac{\Delta E(\theta)}{\sqrt{\Delta E(\theta)^{2}+4 N g_{\mathrm{c}}^{2}}}\right) \\
|C(\theta)|^{2} & =|\cos \phi|^{2}=\frac{1}{2}\left(1-\frac{\Delta E(\theta)}{\sqrt{\Delta E(\theta)^{2}+4 N g_{\mathrm{c}}^{2}}}\right) .
\end{aligned}
$$

Polariton Quantum Dynamics Simulation. To simulate the corresponding polariton quantum dynamics and PL spectra, we use an extended MeanField Ehrenfest approach, where the nuclear DOFs are evolved classically, while electron-photon subsystem is treated quantum mechanically. We use the electronic-Fock basis $\{|u, n\rangle \equiv|u\rangle \otimes|n\rangle\}$, where $|u\rangle \equiv\left\{|G\rangle,\left|X_{i}\right\rangle,\left|\tilde{X}_{i}\right\rangle\right\}$ represents the electronic basis and $|n\rangle=\{|0\rangle,|1\rangle, \ldots\}$ represents vacuum's Fock states. In this study, we only consider the Hilbert subspace $\left\{|G, 0\rangle,|G, 1\rangle,\left|X_{i}, 0\right\rangle,\left|\tilde{X}_{i}, 0\right\rangle\right\}$, because $\left|X_{i}, 1\right\rangle$ and $\left|\tilde{X}_{i}, 1\right\rangle$ are energetically detuned from the rest of the states. In addition to the above unitary time-evolution governed by $\hat{H}_{\mathrm{pl}}$ and $\hat{H}_{\mathrm{L}}$, we also consider the following nonradiative and radiative decays channels: (i) the cavity loss from $|G, 1\rangle$ to $|G, 0\rangle$ with a decay rate $\gamma_{\mathrm{c}}$, (ii) the non-radiative decay from $\left|\tilde{X}_{i}\right\rangle$ to $\left|X_{i}\right\rangle$ within the $i_{\text {th }}$ NPL with a decay rate of $\gamma_{\tilde{x}}$, and (iii) the radiative decay from $\left|E_{i}\right\rangle$ to $|G\rangle$ with a decay rate of $\gamma_{x}$. The details of the quantum dynamics simulation are provided in the Supporting Information.

The reduced density matrix in the electronic-Fock basis is obtained as $\hat{\rho}(t)=\mathbf{c}^{\dagger}(t) \cdot \mathbf{c}(t)$. The reduced density matrix in the polaritonic basis, the eigen-state representation of $\hat{H}_{\mathrm{pl}}(\mathbf{R})$, is obtained as $\hat{\rho}_{\mathrm{pl}}(t)=\hat{U}^{\dagger}(\mathbf{R}) \hat{\rho}(t) \hat{U}(\mathbf{R})$, where $\hat{U}(\mathbf{R})$ diagonalizes $\hat{H}_{\mathrm{pl}}(\mathbf{R})$, such that $\hat{U}^{\dagger}(\mathbf{R}) \hat{H}_{\mathrm{pl}}(\mathbf{R}) \hat{U}(\mathbf{R})=$ $\sum_{\alpha} E_{\alpha}(\mathbf{R}, \theta)|\alpha(\mathbf{R}, \theta)\rangle\langle\alpha(\mathbf{R}, \theta)|$. The PL spectrum is obtained by using $\hat{\rho}^{\mathrm{pl}}(t)$ under the steady-state limit with the following expression, ${ }^{23,24}$

$I_{\mathrm{PL}}(E, \theta)=\left\langle\sum_{\alpha} \frac{\left\langle\alpha\left|\hat{\rho}_{\mathrm{pl}}\left(t_{\mathrm{s}}\right)\right| \alpha\right\rangle \cdot|\langle\alpha \mid G, 1\rangle|^{2} \cdot\left(\Gamma_{\alpha} / 2\right)}{\left(E-E_{\alpha}(\mathbf{R}, \theta)\right)^{2} / \hbar^{2}+\left(\Gamma_{\alpha} / 2\right)^{2}}\right\rangle$,

where $\langle\ldots\rangle$ denotes quantum mechanical average, which is approximated by the trajectory-average in the current mixed quantum-classical simulation, and we use $|\alpha\rangle \equiv|\alpha(\mathbf{R}, \theta)\rangle$ for a short-hand notation, $t_{\mathrm{s}}$ represents the steady-state time limit when polariton population $\left\langle\alpha\left|\hat{\rho}_{\mathrm{pl}}(t)\right| \alpha\right\rangle$ reaches to a steady state (plateau). Further, $\gamma_{\alpha}$ is the radiative decay rate of the polaritonic state $|\alpha(\mathbf{R}, \theta)\rangle$. Because that $\gamma_{c} \gg \gamma_{x}, \Gamma_{\alpha}$ can be approximated as $\Gamma_{\alpha} \approx|\langle\alpha \mid G, 1\rangle|^{2} \gamma_{\mathrm{c}}$. The simulation protocol for PL is provided in the Supporting Information.

Results and Discussions. Fig. 3 a presents the angleresolved reflectance spectrum for a cavity containing a PMMA/NPLs layer. The sample is probed by a broadband white light source. Fig. 3a clearly shows two separated branches in the angle-resolved spectrum, which are identified as the upper polariton (UP) and the lower polariton (LP) branch. The two polariton branches can be fitted to $E_{+}(\theta)$ and $E_{-}(\theta)$ expressed in Eq. 10 . The fitting parameters are the vertical Franck-Condon exciton energy $E_{x}^{0}=E_{x}+\lambda$, the photon energy at zero incident angle $E_{\mathrm{ph}}=\hbar \omega_{\mathrm{c}}$ and the effective coupling strength $\sqrt{N} g_{\mathrm{c}}$. The coupling strength is estimated to be $\sqrt{N} g_{\mathrm{c}}=41.5 \mathrm{meV}$, corresponding to a $\hbar \Omega_{\mathrm{R}}=83$ meV Rabi splitting at the zero detuning $\Delta E\left(\theta_{0}\right)=0$. 
The zero-angle cavity-exciton detuning is estimated to be $\Delta E=-130 \mathrm{meV}$. We further analyze the polaritonic characters of these branches based on the above extracted parameters.
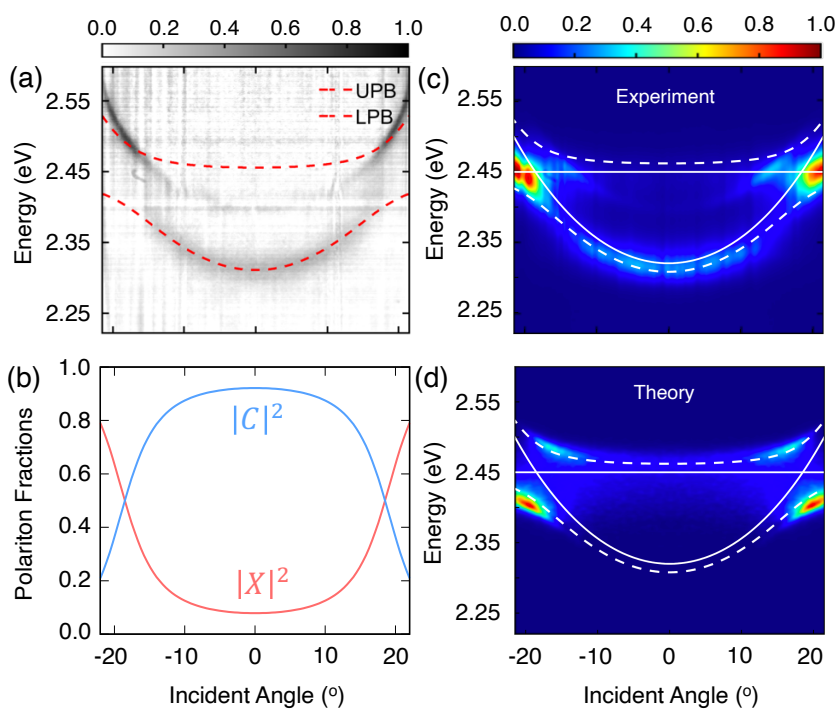

Figure 3: (a) Angle-resolved white light reflectance spectrum of the NPL-cavity hybrid system. (b) The Hopfield coefficients (Eq. 16) of the excitonic $|X|^{2}$ and photonic $|C|^{2}$ fraction extracted from the reflectance spectrum. (c) Experimentally measured angled-resolved photoluminescence spectrum. (d) Angle-resolved photoluminescence spectrum obtained from theoretical simulations. In (a), (c) and (d) dashed lines indicate the upper and lower polariton branches. The solid lines in (c) and (d) indicate the photonic and excitonic branches.

Fig. $3 \mathrm{~b}$ presents the character of the lower polariton state through the Hopfield coefficients $|X|^{2}$ and $|C|^{2}$ which represent the excitonic and the photonic characters, respectively. Since the detuning is large at $\theta=0^{\circ}$, the lower polariton branch is predominantly photonic in character $(\approx 93 \%)$ at low angles, indicated by the red curve in Fig. $3 b$ which remains flat for small incident angles (for less than $\pm 10^{\circ}$ ). On the other hand, at high incident angles (especially $>19^{\circ}$ ) the upper and lower polaritons switch their characters, and the lower polariton becomes predominantly excitonic. At $\approx 19^{\circ}$ the Hopfield coefficients become exactly 0.5 , indicating a resonance condition (zero detuning) between the photonic and excitonic branches.

Fig. 3c presents angle-resolved PL measurements, where the measurements are conducted at $T=8 \mathrm{~K}$. The sample is off-resonantly pumped via a $405 \mathrm{~nm}$ diode laser that couples the ground state and continuous exciton states $\left\{\left|\tilde{x}_{i}\right\rangle\right\}$ of the NPLs (see Eq. 3), and the PL is collected by the angle-resolved spectroscopy system depicted in Fig. 1a. The polariton emission primarily occurs in the lower polariton branch, with a much lower PL intensity from the upper polariton branch. The LP branch flattens as the collection angle increases, vastly deviating from the cavity photon dispersion (white solid lines in Fig. 3c) and moving towards the exciton dispersion (white dashed lines in Fig. 3c), indicating a strong hybridization between the exciton and the photon. Furthermore, the intensity distribution of the lower polariton in Fig. $3 \mathrm{c}$ is significantly different from the angle-resolved reflectance spectrum. Namely, in the LP branch of Fig. 3c, the intensity is congregating at large angle $\left(\approx 19^{\circ}\right)$ while the reflectance intensity at the LP branch at large angles $\left(\approx 19^{\circ}\right)$ is negligible. This asymmetrical intensity of PL agrees with the recent experiments implementing NPLs in a metal Fabry-Perot cavity ${ }^{17,25}$ as well as theoretical simulations. ${ }^{26,27}$

Fig. 3d presents PL spectra obtained by computing Eq. 17 with a mixed quantum-classical dynamics simulation in a model Hamiltonian (Eq. 2). As can be seen, the simulated PL spectra qualitatively as well as semi-quantitatively capture the basic features observed in Fig. 3c, including the congregation of the PL intensity at large angles as well as its asymmetric intensity distribution between lower and upper polariton. In Eq. 17, the PL intensity depends on the following three key factors: (i) the steady-state populations $\left\langle\alpha\left|\hat{\rho}_{\mathrm{pl}}\left(t_{\mathrm{s}}\right)\right| \alpha\right\rangle$ of the polariton branch $|\alpha(\mathbf{R}, \theta)\rangle$, which are significantly influenced by the phonon-mediated non-adiabatic transitions and cavity radiative loss; (ii) the angle-dependent photonic character $|\langle\alpha \mid G, 1\rangle|^{2}$ of a polariton branch, which depends on the detuning $\Delta E(\theta)$ and the light-matter coupling strength $\sqrt{N} g_{\mathrm{c}}$; and (iii) the radiative lifetime $\Gamma_{\alpha}$ which originates from the cavity loss and therefore also depends on the photonic character of the $\alpha_{\text {th }}$ polariton branch. Below we analyze the polaritonic population dynamics at two representative incident angles.

Fig. 4 presents polariton quantum dynamics obtained from our theoretical simulations at two incident angles for the NPL-cavity hybrid system presented in Fig. 3. At a small incident angle, the lower polariton branch is highly detuned from the excitonic manifold. This large detuning can be seen in Fig. 4a, which presents the time-dependent polaritonic energies at $0^{\circ}$ incident angle on a representative phonon trajectory, color coded to depict the instantaneous photonic character as the phonon DOFs evolve in time. Note that the timedependent fluctuations of the polaritonic energies are caused by the phonon DOF. As expected, most of the photonic character $(\approx 93 \%)$ is concentrated at the lower polariton branch. The high-lying polaritonic states have predominant excitonic charactor and only a tiny amount 

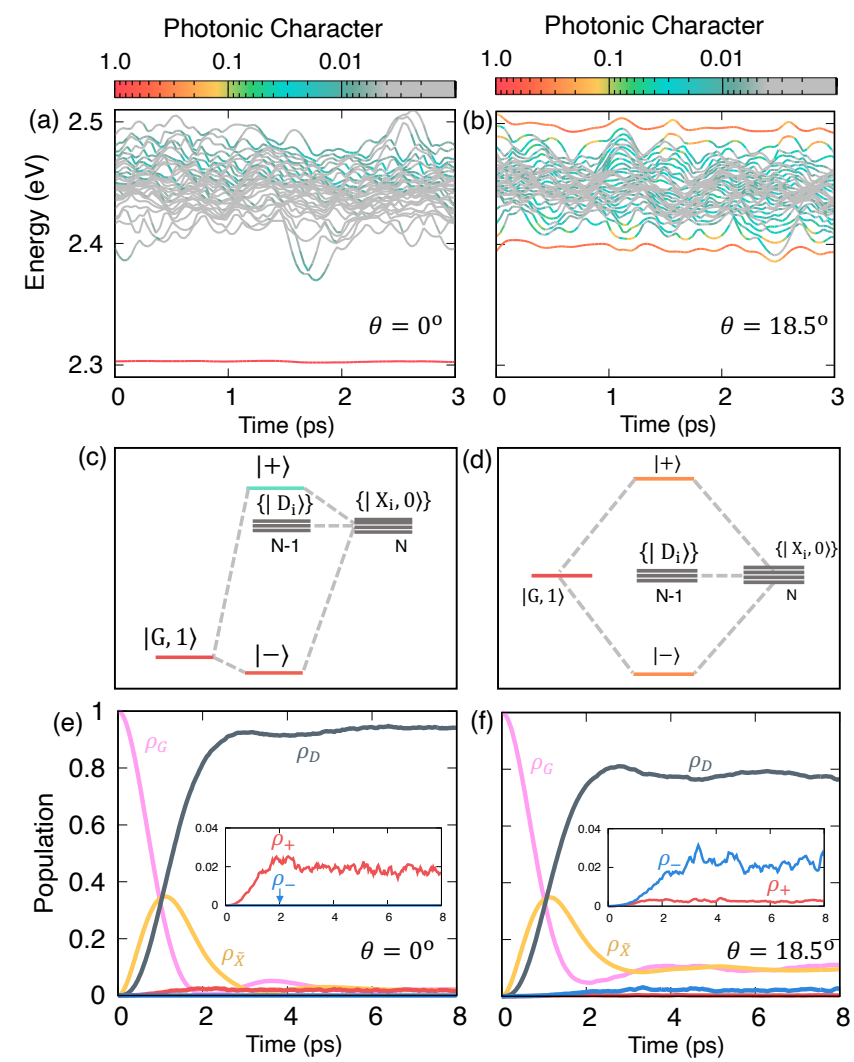

Figure 4: Quantum dynamics of nanoplatelets coupled to the cavity. Time-dependent polaritonic energy for a representative trajectory obtained with numerical simulation with (a) $\theta=0^{\circ}$ and (b) $\theta=18.5^{\circ}$ with all other parameters same as Fig. 3. Panel (c) and (d) present the polariton eigenspectrum that corresponds to (a) and (b), based on Eq. 10 without considering any phonon fluctuations. Time-dependent polaritonic populations of the ground-state ( $\rho_{G}$ in pink), $| \pm\rangle$ states ( $\rho_{ \pm}$in red and blue respectively), laser driven highlying excitonic states ( $\rho_{\tilde{X}}$ in black) as well as the population of the dark-state ( $\rho_{D}$ in black) at (e) $\theta=0^{\circ}$ and (f) $\theta=18.5^{\circ}$.

of photonic character is distributed among them.

Fig. $4 \mathrm{~b}$ presents the time-dependent polaritonic energies at the $18.5^{\circ}$ incient angle on a representative phonon trajectory, color coded according to their photonic character. At this angle, the light-matter detuning is almost zero. Despite the phonon induced fluctuations, both the lower and the upper polariton branches contains a large photonic character $(\approx 40 \%)$. A clear feature arising from thermal fluctuations of phonon is that the upper and lower polariton states can get close to the manifold of dark states that lie in the gap between the upper and lower polariton branches. This behavior is commonly encountered in non-adiabatic dynamics of photo-chemistry, where the fluctuating nuclei enables avoided crossing in electronic adiabatic states. Here, we observe similar behavior of the phonon induced avoid crossing and change of state character in polariton states.

In the absence of the phonon fluctuations, the Holstein-Tavis-Cummings Hamiltonian becomes the well-known Tavis-Cummings model in quantum optics. The photon-dressed states and polariton states for the TC models are presented in Fig. 4c-d, corresponding to the quantum dynamics presented in panel (a) and (b), respectively. In Fig. 4c-d, only the upper and the low polariton branches are expected to possess the photonic character, while the rest of the polaritonic states $(N-1$ degenerate states) are pure excitonic states without any photonic character, as commonly referred to as the "dark states" in the polariton chemistry literature. ${ }^{6,28-30}$ This is because that in the TC model (without the presence of the phonon DOF), the symmetry of the Hamiltonian allows one to express the dressed excitonic states $\left\{\left|X_{i}, 0\right\rangle\right\}$ in a new orthonormal basis $\left\{|B\rangle,\left|D_{k}\right\rangle\right\}$, where $|B\rangle=\frac{1}{\sqrt{N}} \sum_{i}\left|X_{i}, 0\right\rangle$ is called the bright excitonic state. This implies that the rest of the $N-1$ states $\left|D_{k}\right\rangle=\sum_{i} c_{i}^{k}\left|X_{i}, 0\right\rangle$ which follows the orthogonality relation $\left\langle B \mid D_{k}\right\rangle=0$ will satisfy $\sum_{i} c_{i}^{k}=0$. As a result, only $|B\rangle$ couples to the $|G, 1\rangle$ with a scaled coupling $\left\langle B\left|\hat{H}_{\mathrm{pl}}\right| G, 1\right\rangle=\sqrt{N} g_{\mathrm{c}}$ which leads to the formation of $| \pm\rangle$ states. On the other hand, $\left|D_{k}\right\rangle$ states are strictly decoupled (hence are referred to as the dark states) from the $|G, 1\rangle$ state as $\left\langle D_{k}\left|\hat{H}_{\mathrm{pl}}\right| G, 1\right\rangle=g_{\mathrm{c}} \sum_{i} c_{i}^{k}=0$. While this simplified picture shown in Fig. $4 \mathrm{c}-\mathrm{d}$ has been commonly used to understand polariton photophysics, it is no longer a strictly valid picture in a realistic molecule cavity hybird system where the presence of the phonon fluctuations will make the dark states have some photonic character, as shown in Fig. 4a-b. In particular, the dark states are no longer strictly dark, but will have photonic contributions and mediate the population transfer through phonon induced avoid crossings among the polaritonic states $|\alpha(\mathbf{R}, \theta)\rangle$.

Fig. $4 \mathrm{e}$ presents the polariton population dynamics at an incident angle of $0^{\circ}$. The NPL-Cavity hybrid system is initiated at $|G, 0\rangle$ which is the ground state of NPLs with zero photons in the cavity. This state is also the ground state of the HTC model Hamiltonian. Upon turning on the CW laser excitation at $t=0$, the higher-lying excited states of the NPLs start to get populated. As a result, the population $\rho_{G}$ of state $|G, 0\rangle$ (pink solid line) in Fig. 4e decreases as the total population of the high-lying NPL states $\rho_{\tilde{X}}=\sum_{i} \rho_{\tilde{X}_{i}}$ starts to increase (yellow solid line) at short-times for $t<1$ ps. Due to the non-radiative transition from the high-lying NPL states $\left\{\left|\tilde{X}_{i}, 0\right\rangle\right\}$ to the excitonic states $\left\{\left|X_{i}, 0\right\rangle\right\}$, the total dark-state population $\rho_{D}=\sum_{k} \rho_{D_{k}}$ as well as the population of upper polariton branch $\rho_{+}$(which is pre- 
dominantly excitonic) grows up till $\mathrm{t}=2 \mathrm{ps}$ as it reaches steady-state. Noticeably, the lower polaritonic $\rho_{-}$population remains negligible throughout. There are several factors that lead to this negligible population in the $|-\rangle$ state. First, due to low excitonic character of $|-\rangle$, non-radiative population transfer from high-lying laser-driven excitonic states $\left\{\left|\tilde{X}_{i}, 0\right\rangle\right\}$ to the $|-\rangle$ state is substantially low. Second, due to large photonic character of the the $|-\rangle$ state, the rate of the radiative losses from this state is also high. Third, since $|-\rangle$ is energetically far away from the $|+\rangle$ state or the dark states (as can be seen in Fig. 4a), the phonon fluctuations induced non-adiabatic transitions to the $|-\rangle$ state are also negligible. All of these factors result in a very low steadystate population for the $|-\rangle$ state. Due to this, at the low incident angles the PL intensity of the lower polariton branch is negligible, despite that LP has a relatively high photonic character as shown in Fig. 3a. On the other hand, while steady-state populations for the rest of the polariton branches are comparatively larger than the $|-\rangle$ state, these polariton branches do not show up in the PL spectra at low angles due to their negligible photonic character. This explains the low intensities of PL signals for both branches of polariton states at lower incident angles (at high detuning).

Fig. 4f presents the population dynamics of the NPLcavity system in Fig. 3 at the incident angle of $18.5^{\circ}$. Here, the $|-\rangle$ state is more populated than the $|+\rangle$ state, a situation opposite to the case in Fig. 4e. With a nearly zero light-matter detuning, both the $|+\rangle$ and $|-\rangle$ states have nearly equal photonic or excitonic character (as shown in Fig. 4b). This equality arises from the fact that polaritonic energies are closely packed, and the phonon-induced non-adiabatic transitions result in population transfers from the upper polariton to the dark states and eventually to the lower polariton. These results explain the observed asymmetry in the steady state populations between $|+\rangle$ and $|-\rangle$ state. Consequently, this asymmetry of the polariton population manifests itself into the PL spectra (Eq. 17) in Fig. 3c-d. Therefore, phonon assisted population transfer plays an essential role in the NPL-cavity systems especially around zero detuning. Overall, our simulations suggest that the congregation of the PL intensity at a higher incident angle is due to an interplay among (i) phonon-mediated nonadiabatic transitions between polaritons, (ii) cavity loss, and (iii) angle-dependent photonic character of the polariton branches.

From the above experiments and theoretical simulations, we find that at smaller detunings, phonon induced non-adiabatic transitions play a crucial role in depleting the upper polaritonic steady state population and trans- ferring population to the lower polariton, due to nonadiabatic transition from the "dark-states" (Fig. 4b and f). Meanwhile, with a large negative detuning (Fig. 4a and e), the excitonic branches are nearly decoupled from the photonic branch. In such cases, while the excitonic branches have relatively large steady state populations due to non-radiative transitions from high-lying excitonic states $\left(\left|\tilde{X}_{i}\right\rangle \rightarrow\left|X_{i}\right\rangle\right)$, they have negligible photonic character, making them non-emissive. In a similar scenario, the photon-dominated branch (upper branch for positive detuning or lower branch for negative detuning) will not accumulate an appreciable amount of steady state population, leading to a weak PL intensity. Overall, it is expected to observe significant population in the vicinity of zero angle in the lower polariton branch only at small detunings.

In Fig. 5, we further verify our theoretical interpretation by making three NPLs-cavity devices with different detunings at the zero incident angle, while keeping the light-matter coupling strength at the same level $\left(\hbar \Omega_{R} \approx 62 \sim 71 \mathrm{meV}\right)$. In Fig. 5a, the detuning $\Delta E=$ $-15.7 \mathrm{meV}$ is smaller than the device used in Fig. 3. Therefore, the photonic and excitonic branches become resonant at smaller angles than the device in Fig. 3, leading to a high intensity in the lower polariton branch at small incident angles. Further, in Fig. 5b the detuning is increased to $\Delta E=-29.6 \mathrm{meV}$, where the resonance occurs at relatively higher incident angles $\left(\approx \pm 10^{\circ}\right)$ than in Fig. 5a. Due to this, polariton population congests at a higher angle. Finally, Fig. 5c presents the PL spectra at an even higher detuning with $\Delta E=-34.6$ $\mathrm{meV}$ at zero-angle. Following the same trend, as the exciton-photon resonance occurs at an even higher angle $\left(\approx \pm 14^{\circ}\right)$ and the PL intensity are further separated into two clusters compared to Fig. 5 b.

On top of the qualitative prediction of the PL intensity, our theoretical simulations in Fig. 5d-f is commensurate with the experimentally observed PL in Fig. 5a$\mathrm{c}$, including the asymmetric PL intensity of the upper and lower polariton branches, as well as an increase of the lower polariton PL peak separation with increased exciton-photon detunings. All of these agree with our theoretical picture of the phonon-assisted nonadiabatic transitions among polaritonic states at various incident angles. However, there are limitations of our current theoretical approach, including treating the incoming momentum (incident angle) of the photon as a parameter (instead of a dynamical variable). These theoretical limitations, together with detailed plans to address them, are discussed in the Supporting Information. That said, the excellent agreement in Fig. 3 and Fig. 5 demonstrates that our current theoretical ap- 

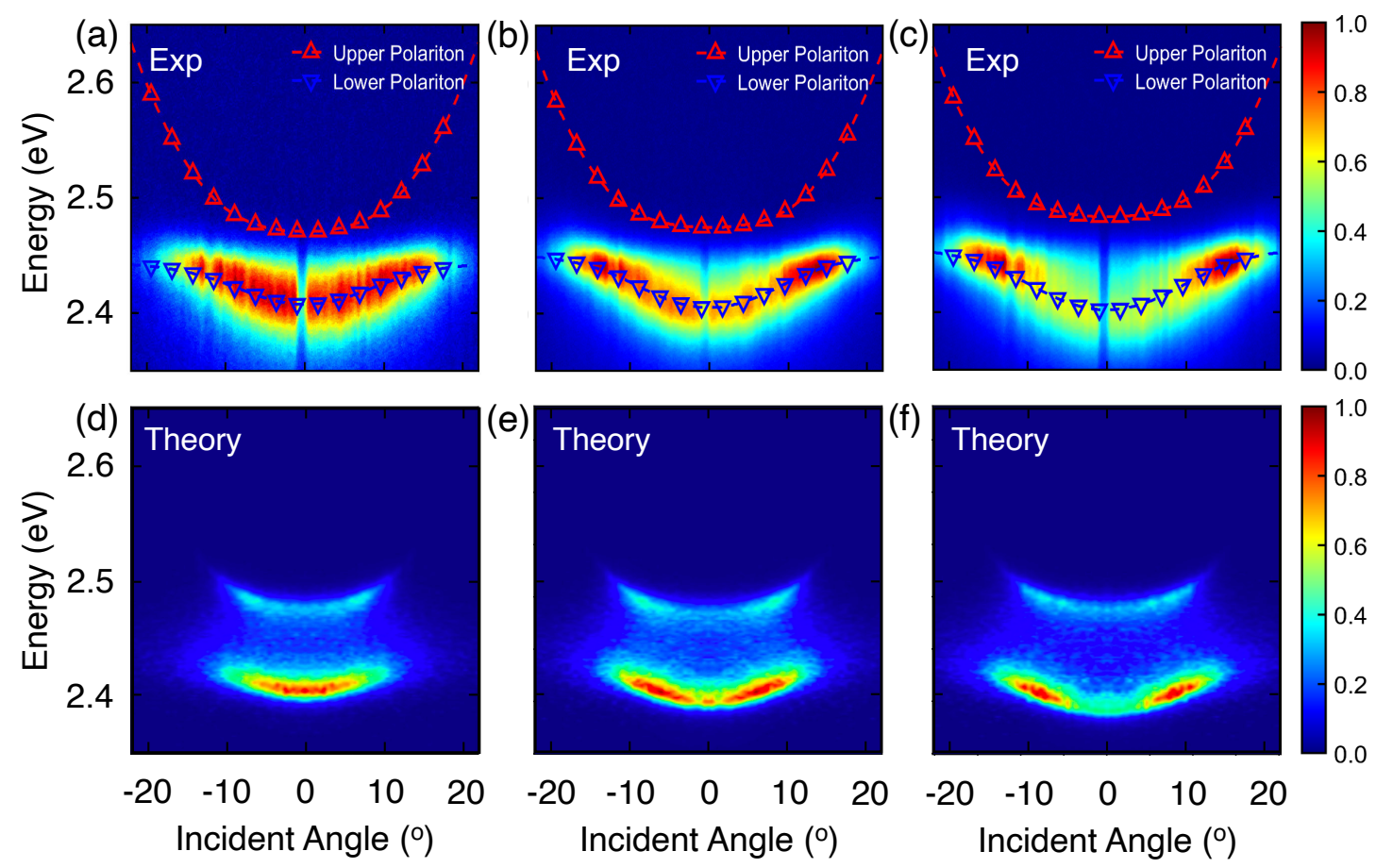

Figure 5: Angle resolved photoluminescence spectra for the NPL-cavity hybrid system obtained experimentally with a range of different detuning $\Delta E$ and Rabi splitting $\hbar \Omega_{\mathrm{R}}$ : (a) $\Delta E=-15.7 \mathrm{meV}, \Omega_{\mathrm{R}}=61.1 \mathrm{meV}$; (b) $\Delta E=-29.6 \mathrm{meV}$, $\hbar \Omega_{R}=$ $62.9 \mathrm{meV}$; and (c) $\Delta E=-34.6 \mathrm{meV}, \hbar \Omega_{R}=73 \mathrm{meV}$. Angle resolved photoluminescence spectra obtained with theoretical simulations in (d)-(f) correspond to those experimental results presented in (a)-(c), respectively.

proaches capture the essential polariton photophysics in the NPL-cavity hybrid systems.

Conclusions. In this work, we demonstrate strong light-matter coupling between CdSe NPL excitons and the photonic states of a dielectric optical cavity. We characterize the polariton states with angle-resolved reflectance and photoluminescence measurements, suggesting a Rabi splitting as large as $83 \mathrm{meV}$, comparable to that obtained in previously reported NPL-metal cavity hybrid systems. ${ }^{17,18,21}$ We perform numerical simulations to obtain angle-resolved PL spectra via a mixed quantum-classical dynamics approach. The PL intensity from a particular polariton branch depends on the interplay between its steady-state population, photonic character and radiative losses. Our combined theoretical and experimental results indicate that at a small exciton-photon detuning, the phonon assisted nonadiabatic transitions between polariton states play a crucial role in depleting the population in the upper polariton branch and transferring population to the lower polariton branch. Meanwhile, at a larger detuning, the PL intensity from both upper and lower branches diminishes as the photon-dominated branch is not significantly populated while the exciton-dominated branch lacks sufficient photonic character to emit. We further confirm our theoretical interpretation with three additional NPL-cavity systems with different zero-angle detunings.

Overall, our work provides a comprehensive mechanistic understanding of the polariton photophysics in an NPLs-cavity hybrid system and demonstrates the feasibility of strong couplings between NPLs with the quantized radiation in a cavity. The system demonstrated here has the potential for investigating polariton mediated charge transfer ${ }^{31-34}$ where the cavity can significantly influence the photo-induce charge transfer reaction from NPLs to acceptor molecules such as methyl viologen. ${ }^{35}$ In a separate direction, our current investigation will pave the way toward to explore the transduction of quantum states among various physical degrees of freedom (electronic, vibronic, polaritonic), providing the emerging quantum information science field the knowledge for preparing next generation quantum systems.

\section{Acknowledgments}

This work was supported by the National Science Foundation "Enabling Quantum Leap in Chemistry" program under Grant numbers CHE-1836566 and CHE-1836546. L. Q. and 
A. N. V acknowledge the support from the Air Force Office of Scientific Research under Grant FA9550-19-1-0074 for supporting initial cavity fabrication. P. H. acknowledges the support from a Cottrell Scholar award (a program by Research Corporation for Science Advancement). Computing resources were provided by the Center for Integrated Research Computing (CIRC) at the University of Rochester.

\section{References}

(1) Hutchison, J. A.; Schwartz, T.; Genet, C.; Devaux, E.; Ebbesen, T. W. Modifying Chemical Landscapes by Coupling to Vacuum Fields. Angew. Chem. Int. Ed. 2012, 51, 1592-1596.

(2) Schwartz, T.; Hutchison, J. A.; Genet, C.; Ebbesen, T. W. Reversible Switching of Ultrastrong Light-Molecule Coupling. Phys. Rev. Lett. 2011, 106, 196405.

(3) Munkhbat, B.; Wersall, M.; Baranov, D. G.; Antosiewicz, T. J.; Shegai, T. Suppression of PhotoOxidation of Organic Chromophores by Strong Coupling to Plasmonic Nanoantennas. Sci. Adv. 2018, 4, eaas9552.

(4) Stranius, K.; Hertzog, M.; Börjesson, K. Selective Manipulation of Electronically Excited States Through Strong Light-Matter Interactions. Nat. Commun. 2018, 9, 2273.

(5) Kowalewski, M.; Mukamel, S. Manipulating Molecules with Quantum Light. Proc. Natl. Acad. Sci. U.S.A. 2017, 114, 3278-3280.

(6) Feist, J.; Galego, J.; Garcia-Vidal, F. J. Polaritonic Chemistry with Organic Molecules. ACS Photonics 2018, 5, 205-216.

(7) Mandal, A.; Huo, P. Investigating New Reactivities Enabled by Polariton Photochemistry. J. Phys. Chem. Lett. 2019, 10, 5519-5529.

(8) Ebbesen, T. W. Hybrid Light-Matter States in a Molecular and Material Science Perspective. Acc. Chem. Res. 2016, 49, 2403-2412.

(9) Thomas, A.; Lethuillier-Karl, L.; Nagarajan, K.; Vergauwe, R.; J. George, T. C.; Shalabney, A.; Devaux, E.; Genet, C.; Moran, J.; Ebbesen, T. Tilting a Ground-State Reactivity Landscape by Vibrational Strong Coupling. Science 2019, 363, 615-619.
(10) Ribeiro, R. F.; Martínez-Martínez, L. A.; Du, M.; Campos-Gonzalez-Angulo, J.; Yuen-Zhou, J. Polariton Chemistry: Controlling Molecular Dynamics With Optical Cavities. Chem. Sci. 2018, 9, 6325-6339.

(11) Arnardottir, K. B.; Moilanen, A. J.; Strashko, A.; Törmä, P.; Keeling, J. Multimode Organic Polariton Lasing. Phys. Rev. Lett. 2020, 125, 233603.

(12) Keeling, J.; Kena-Cohen, S. Bose-Einstein Condensation of Exciton-Polaritons in Organic Microcavities. Annu. Rev. Phys. Chem. 2020, 71, 435-459.

(13) Takahashi, S.; Watanabe, K. Decoupling from a Thermal Bath via Molecular Polariton Formation. J. Phys. Chem. Lett. 2020, 11, 1349-1356.

(14) Avramenko, A. G.; Rury, A. S. Quantum Control of Ultrafast Internal Conversion Using Nanoconfined Virtual Photons. J. Phys. Chem. Lett. 2020, 11, 1013-1021.

(15) Ithurria, S.; Dubertret, B. Quasi 2D colloidal CdSe platelets with thicknesses controlled at the atomic level. J. Am. Chem. Soc. 2008, 130, 16504-16505.

(16) Ithurria, S.; Tessier, M.; Mahler, B.; Lobo, R.; Dubertret, B.; Efros, A. L. Colloidal nanoplatelets with two-dimensional electronic structure. Nat. Mater. 2011, 10, 936-941.

(17) Flatten, L. C.; Christodoulou, S.; Patel, R. K.; Buccheri, A.; Coles, D. M.; Reid, B. P.; Taylor, R. A.; Moreels, I.; Smith, J. M. Strong exciton-photon coupling with colloidal nanoplatelets in an open microcavity. Nano Letters 2016, 16, 7137-7141.

(18) Winkler, J. M.; Rabouw, F. T.; Rossinelli, A. A.; Jayanti, S. V.; McPeak, K. M.; Kim, D. K.; Le Feber, B.; Prins, F.; Norris, D. J. Room-temperature strong coupling of $\mathrm{CdSe}$ nanoplatelets and plasmonic hole arrays. Nano letters 2018, 19, 108-115.

(19) Tessier, M. D.; Javaux, C.; Maksimovic, I.; Loriette, V.; Dubertret, B. Spectroscopy of single CdSe nanoplatelets. ACS nano 2012, 6, 67516758.

(20) Steigerwald, M. L.; Brus, L. E. Semiconductor crystallites: a class of large molecules. Acc. Chem. Res. 1990, 23, 183-188. 
(21) Shlesinger, I.; Monin, H.; Moreau, J.; Hugonin, J.-P.; Dufour, M.; Ithurria, S.; Vest, B.; Greffet, J.-J. Strong Coupling of Nanoplatelets and Surface Plasmons on a Gold Surface. ACS Photonics 2019, 6, 2643-2648.

(22) Rossinelli, A. A.; Riedinger, A.; MarquésGallego, P.; Knüsel, P. N.; Antolinez, F. V.; Norris, D. J. High-temperature growth of thick-shell $\mathrm{CdSe} / \mathrm{CdS}$ core/shell nanoplatelets. Chem. Commun. 2017, 53, 9938-9941.

(23) Herrera, F.; Spano, F. C. Absorption and photoluminescence in organic cavity QED. Phys. Rev. A 2017, 95, 053867.

(24) Herrera, F.; Spano, F. C. Dark Vibronic Polaritons and the Spectroscopy of Organic Microcavities. Phys. Rev. Lett. 2017, 118, 223601.

(25) Müller, K.; Fischer, K. A.; Rundquist, A.; Dory, C.; Lagoudakis, K. G.; Sarmiento, T.; Kelaita, Y. A.; Borish, V.; Vučković, J. Ultrafast Polariton-Phonon Dynamics of Strongly Coupled Quantum Dot-Nanocavity Systems. Phys. Rev. X 2015, 5, 031006.

(26) Roy, C.; Hughes, S. Polaron master equation theory of the quantum-dot Mollow triplet in a semiconductor cavity-QED system. Phys. Rev. B 2012, 85,115309 .

(27) Neuman, T.; Aizpurua, J. Origin of the asymmetric light emission from molecular excitonpolaritons. Optica 2018, 5, 1247-1255.

(28) Du, M.; Martínez-Martínez, L. A.; Ribeiro, R. F.; $\mathrm{Hu}, \mathrm{Z}$.; Menon, V. M.; Yuen-Zhou, J. Theory for Polariton-Assisted Remote Energy Transfer. Chem. Sci. 2018, 9, 6659-6669.

(29) Galego, J.; Garcia-Vidal, F. J.; Feist, J. ManyMolecule Reaction Triggered by a Single Photon in Polaritonic Chemistry. Phys. Rev. Lett. 2017, 119, 136001.

(30) Campos-Gonzalez-Angulo, J. A.; Ribeiro, R. F.; Yuen-Zhou, J. Resonant Catalysis of ThermallyActivated Chemical Reactions With Vibrational Polaritons. Nat. Commun. 2019, 10, 4685.

(31) Herrera, F.; Spano, F. C. Cavity-Controlled Chemistry in Molecular Ensembles. Phys. Rev. Lett. 2016, 116, 238301.
(32) Semenov, A.; Nitzan, A. Electron Transfer in Confined Electromagnetic Fields. J. Chem. Phys. 2019, 150, 174122.

(33) Mandal, A.; Krauss, T. D.; Huo, P. PolaritonMediated Electron Transfer via Cavity Quantum Electrodynamics. J. Phys. Chem. B 2020, 124, 6321-6340.

(34) Wellnitz, D.; Pupillo, G.; Schachenmayer, J. A quantum optics approach to photoinduced electron transfer in cavities. J. Chem. Phys. 2021, 154, 054104.

(35) Okuhata, T.; Tamai, N. Face-Dependent Electron Transfer in CdSe Nanoplatelet-Methyl Viologen Complexes. J. Phys. Chem. C 2016, 120, 1705217059. 